\title{
TOWARDS ADAPTIVE ONTOLOGY-BASED \\ VIRTUAL BUSINESS NETWORKS
}

\author{
Peter Weiß \\ Alexander Maedche \\ FZI Forschungszentrum Informatik Karlsruhe, GERMANY, (pweiss, maedche)@fzi.de
}

\begin{abstract}
In the future scenarios of evolving business networks, enterprises will transform themselves into better forms by becoming "more intelligent". "Intelligent" means ability of a network or company to react and adapt to changing market opportunities and conditions. Consequently, a fast and easy docking and quick formation of network entities or new business partners providing needed competencies is vital. In this paper we propose an ontologybased methodology (ODAMY) allowing flexible ontology-based partner integration into existing or evolving business networks. On the basis of an empirical model describing key criteria for successful partner integration, we show how ontologies can be applied to support a fast, on demand integration of required resources. ODAMY will be outlined facilitating partner fit and matching seen as crucial functionalities in future dynamic business ecosystems. Our approach is presented using examples and ODAMY is introduced applying ontologies. ODAMY allows accessing and providing information in machine understandable form.
\end{abstract}

\section{INTRODUCTION}

In the future scenarios of evolving business networks, enterprises will transform themselves into better forms by becoming "more intelligent". These enterprises will be part of some sustainable collaboration networks catalyzing the formation of dynamic virtual organizations to adapt to changing market opportunities and conditions. The networks will form dynamic partnerships with other enterprises to create dynamic business ecosystems, which will be self-managed, self-configured and self-optimized. In this paper we address strategic fit of business partners within virtual organizations and propose an ontology-based framework for business partner integration.

\subsection{Contribution of this paper}

This paper introduces the ontology-based methodology ODAMY'. ODAMY will be outlined facilitating partner fit and matching seen as crucial functionalities in future dynamic business ecosystems. Our approach is applying ontologies as data structure 
and storage mechanism for the enterprise profiles. Ontologies allow to visualize the data structure and underlying conceptual model as well as to enrich queries with semantic information. Furthermore, using ontologies is important regarding machine readability of stored information and data. A reference ontology is modeled by using KAON tool set. As an example we use assessed profiles from a pilot show case. These profiles are stored into the ontology as instances. Consequently, ODAMY allows combining empirical research and semantic web to achieve the aimed flexibility and adaptability through on demand business partner integration. Basic idea is to represent assessed questionnaire entries as instances in the ontology. Thus, we achieve a machine readable and understandable description of business network entities. Thereby, behavior and structure of business networks is analyzed to develop future reference models. Furthermore, we achieve enhanced agility, flexibility and efficiency for existing business networks. Therefore, our research is focused on the ontology-based integration of new business partners and the management of synergies by provision of semantically-enriched information.

\section{MOTIVATION}

\subsection{Integration of new partners}

In figure 1 the partner integration process from an open-ended collection of prequalified partners is shown. The partners agree to form a pool of potential members of virtual organizations. These potential members are selected from the universe of modules. The universe of modules is the set of all organizations and is composed of the individual organizations of, for example, a specific industry or economy. While in the virtual organization, the objective is to exploit a specific market opportunity, the purpose of the dynamic web is a disposition to work together in a future market opportunity.

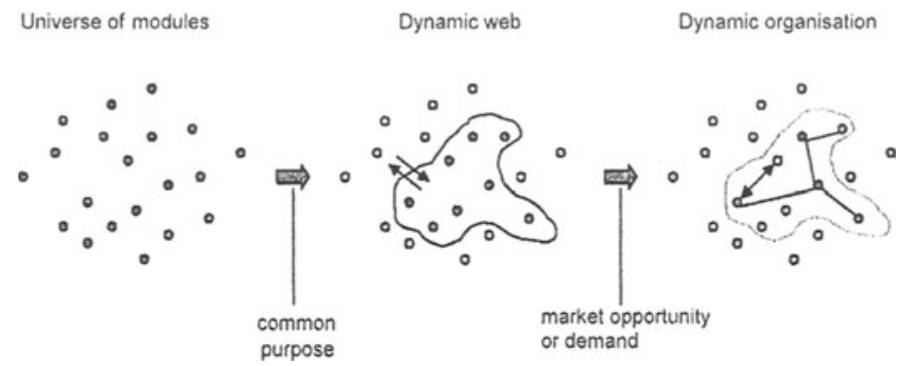

Figure 1: Dynamic formation of new partners and partner fit (W. Saabeel, T.M. Verduijn, L. Hagdorn, K. Kumar, 2002)

The on demand integration of new partners in such a network is in practice a difficult and time consuming task which runs contrary to the basic idea of flexibility and anticipated fast reactions for virtual organizations $s^{i i}$. The transaction cost theory is manifesting this statement arguing the need to reduce efforts to find, select, negotiate and integrate the right business partners. Our experience is that partner fit is a crucial point for success of virtual organizations. Our investigation of virtual 
organizations determined several criteria in our opinion essential for partner fit and allowing an easy and fast docking of business partners.

\subsection{Managing synergies and partner fit}

Besides of the important task to integrate new partners into an existing network, there is the need to maintain and support the formation of virtual corporations. Fanke/ Hickmann (1999) propose the role of a Net-Broker addressing the need for co-ordination. The Net-Broker is an institutionalized coordinator. He acts as a facilitator and catalyst. Brokers help companies to form strategic partnerships, organize network activities and identify new business opportunities. Their task is to spread the network concepts, promote co-operation, organize groups of firms, and connect them to the product designers, marketing specialists, training providers, and industry services programs they need to compete successfully.

\section{OBJECTIVES}

In this paper we introduce ODAMY as an co-ordination mechanism for virtual organizations. Sieber concludes that five facets should be taken into consideration for establishing the co-ordination mechanism: 1) Creation of a complementary resource base from different organizations; 2) Co-operation of partners by common business understanding; 3) Integration of resource base by transaction governance system; 4) Formulation of a common network strategy; and 5) Socialization by trust among involved partners. Corresponding to the Kluber's wheel of virtual organizing model, the whole life-cycle of the virtual organizations can be separated into four stages: Identify potential, Define strategy, Develop structures, and Co-ordination mechanisms (Su, Chen, Lee 2001 (11)). Our objective is to support the different phases of the VO life cycle. We focus on reduction of efforts and transactions costs for business partner selection and team forming. In this paper we introduce success factors and key criteria for partner fit and integration based on an elaborated and tested empirical model. The empirical model is shown in section five introducing metrics for strategic partner fit. We propose an ontology-based methodology ODAMY for business partner integration and selection. ODAMY implements a netbrokerage functionality, which may support the management - or more visionary will allow to a certain degree automation of business partner selection and team forming. Automation in sense of automated information access based on machineprocessable semantics of data and heuristics that use these meta data.

\section{ONTOLOGY}

For the modeling of the ontology we use a conceptual modeling approach, currently being developed within KAON - a platform for developing and deploying semantics-driven enterprise applications (Maedche, Motik, Volz, 2002). The application of ontologies is increasingly seen as key to enabling semantics-driven information access. An OI-model represents a self contained unit of structured 
information that may be reused. A mathematical definition of the conceptual modeling language can be found in (Motik, Maedche, Volz, 2002).

\subsection{Semantic Web}

The currently evolving Semantic Web promises automated information access based on machine-processable semantics of data and heuristics that use these meta data. Within this "new Web", the explicit representation of the semantics of data, accompanied with domain theories (i.e., ontologies), will enable a web that provides a qualitatively new level of service. It will weave together an incredibly large network of human knowledge and will complement it with machine processability. Various automated services will help the user achieve goals by accessing and providing information in machine understandable form. This process may ultimately create extremely knowledgeable systems with various specialized reasoning services systems that can support us in nearly all aspects of life and that will become as necessary to us as access to electric power.

\subsection{Ontologies}

Ontologies are increasingly seen as a key technology for enabling semantics-driven knowledge processing. They are shared conceptual models established by a community of interest and provide a framework for sharing precise meaning of symbols exchanged in the course of communication (Maedche et al., 2001). Many applications benefit from semantically-enriched information, e.g. knowledge management or e-business, to name just a few. It is widely accepted that nextgeneration knowledge management systems will rely on conceptual models in form of ontologies in order to precisely define the meaning. In KAON ontologies consists of concepts, properties and instances grouped in reusable units called OI-models (ontology-instance models).

\section{ODAMY}

ODAMY combines empirical research and modeling as well as application of ontologies. The methodology supports the coordination tasks and is promising approach for future web-based automation within virtual organizations. Figure 3 depicts our developed methodology for an ontology based partner relationship management (PRM).

\subsection{Knowledge acquisition}

A reference ontology has been modeled mainly based on our empirical model from previous research introduced section five (5). Defined criteria and dimensions to describe business network typology and partner fit are modeled using OI-modeler (see section 6) as reference ontology. Figure 3 depicts the reference ontology showing concepts, properties and instances. The company descriptions (e.g. including competencies, financial figures, ratings, used ICT technologies, etc.) are 
stored as instances. The resulting company profile is machine readable and understandable.

\subsection{End-user application}

For end-user application a web interface is designed offering "query" and "matching" functionalities. The end-user is able to explore the reference ontology as well as send queries to search for information about business partners (e.g. partners, competencies). The data structure is visible for the end-user and search hits are enriched by semantic information. "Matching" is combining query and ranking. For example an end-user sends queries about requirements of possible partners and the gets as result a list of possible partners ranked best fitting to partner profile.

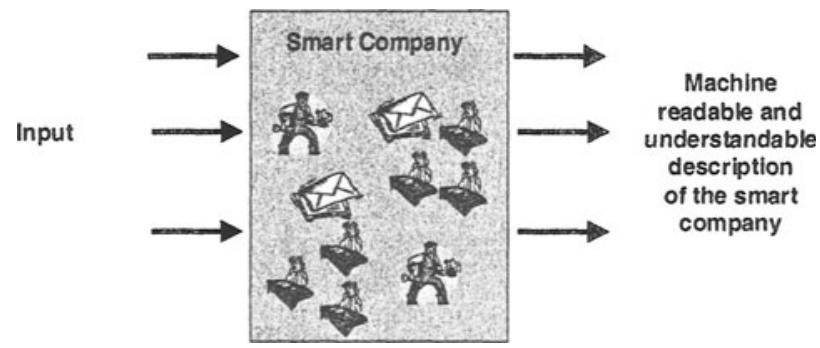

Figure 2: Describing companies profiles formally

\subsection{Analysis}

The analysis package covers two aspects. On the one hand, it includes formal methods and techniques to analyse and continuously improve the reference ontology (mainly based on data-mining techniques). On the other hand, the quality management of registered business partners is supported. The management is able to track the network entities and give performance ratings to identify excellent and bad network performers. The available competencies can be analysed and the portfolio be improved by integrating new partners offering new competencies. In this paper, we present the process of knowledge acquisition.

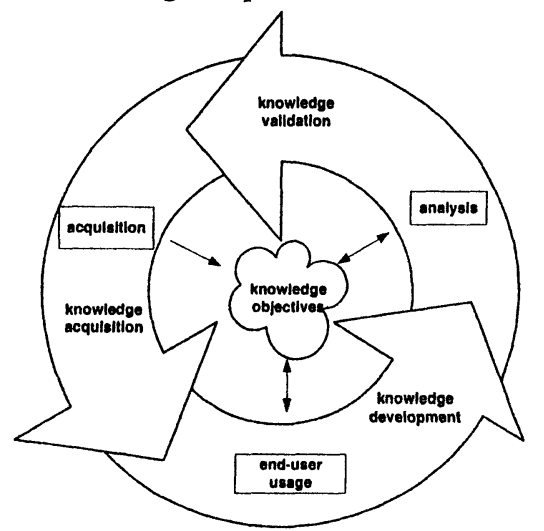

Figure 3: Ontology-based partnership relationship management methodology (ODAMY) 


\section{STRATEGIC FIT}

In this section we introduce our empirical model based on previous research on network typology (inter-net perspective) and strategic partner fit (intra-net perspective).

\subsection{Inter-net perspective}

Seven dimensions (flexibility, time-bonding, trust, market, need of integration, intensity of linkage, network structure) were condensed into four coefficients: (1) degree of centralisation, (2) intensity of linkage, (3) flexibility and (4) confidence. Based on an empiric study the companies could be categorised into different clusters.

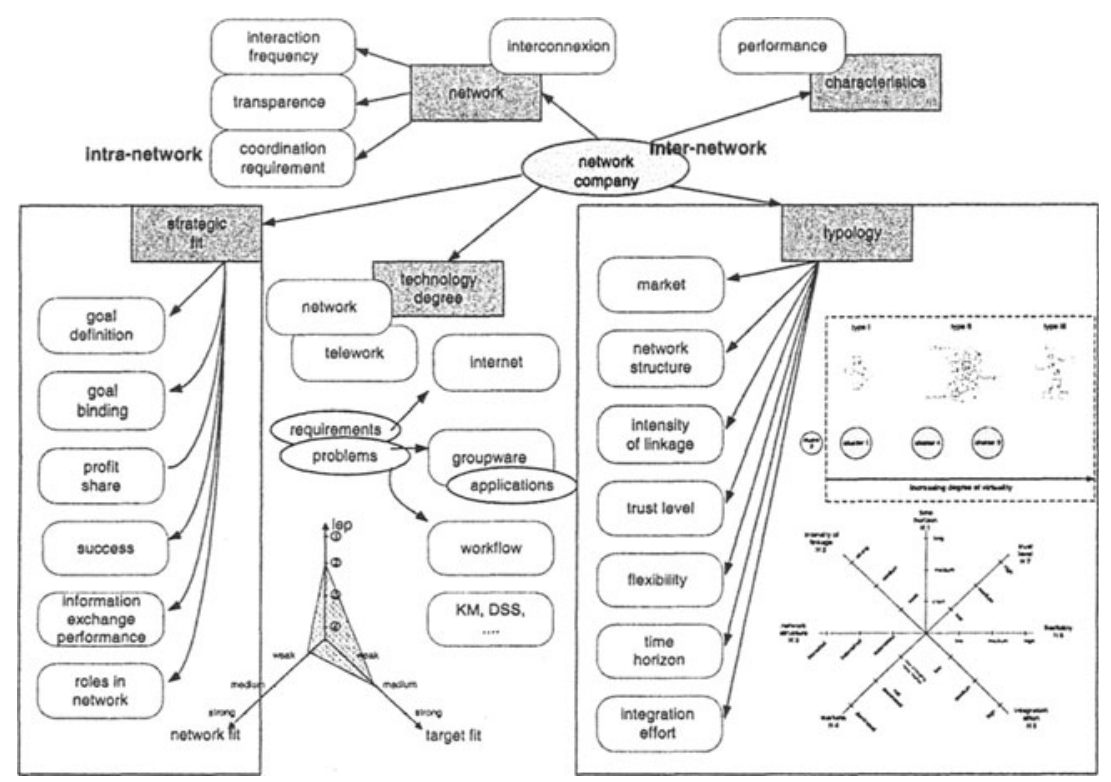

Figure 4: Dimensions to measure network typology and strategic fit

\subsection{Intra-net perspective}

The quality of strategic fit of business partners in the network is measured by three dimensions: (1) target fit, (2) network fit and (3) information exchange performance (iep). The target fit, showing whether the objectives of the individual company are congruent to the network objectives. The network fit measures how strong the success of the company is relied to the success of the network. Due to the lack of formal infrastructures, communication and the exchange of information within the network is of major importance. We distinguish between the request and the 
circulation of information. To be able to scale the iep, internal and external iep have to be distinguished.

\section{ONTOLOGY}

In figure 5 the empirical model is shown as conceptual model for a virtual organization in form of ontology. The figure depicts modelled concepts and properties. The properties are describing the relationship between the different concepts. A network company is described regarding strategic fit, network typology, applied ICT technologies and company characteristics as outlined in figure 4. As additional values for the strategic management of the business network, qualitative and quantitative information can be added to a company representing for example the quality of business performance within the network. ODAMY allows continuously adjusting and expanding the applied empirical model by new indicators. Thereby, reference models may be developed and transformed into shared conceptual models established by a community of interest. The data is assessed by means of a questionnaire.

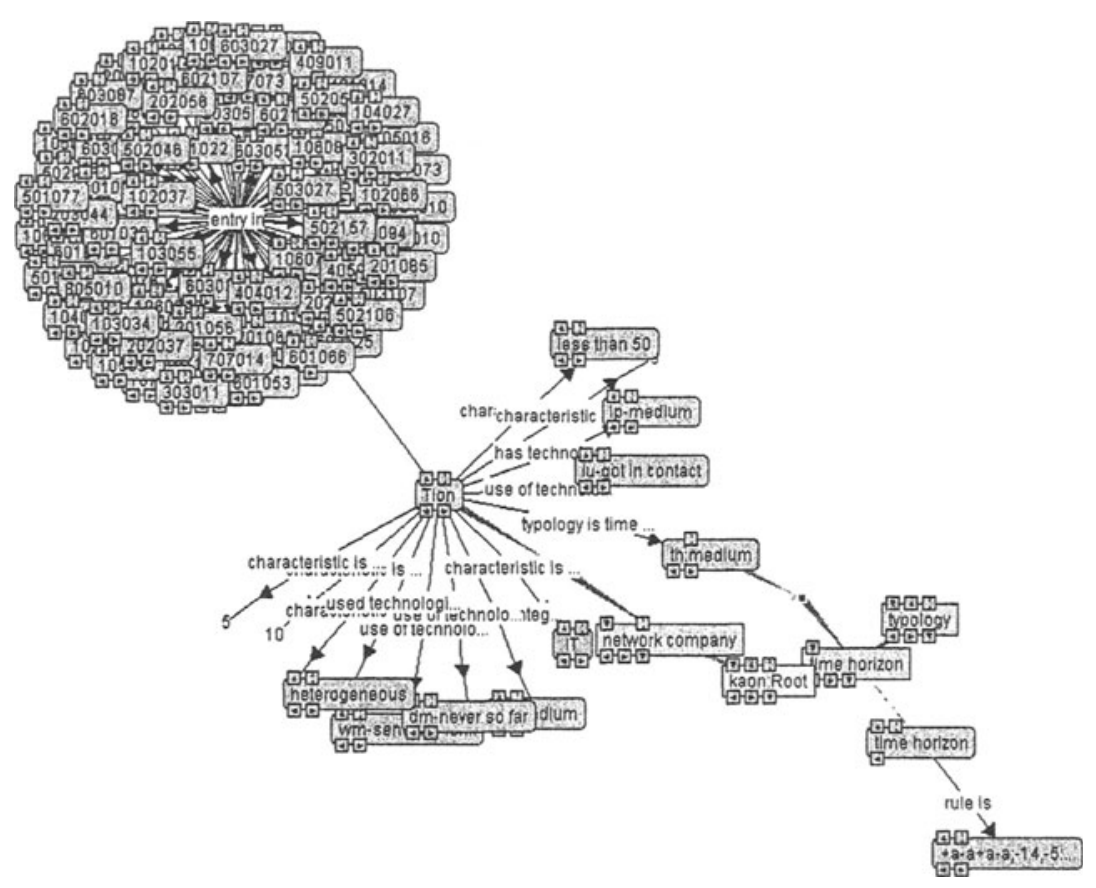

Figure 5: Transformation from questionnaire value entries into ontology instances for company description

The questionnaire value entries are represented as instances of the company ontology. Figure 5 depicts questionnaire value entries after transformation into ontology instances. ODAMY allows querying for the best fitting company in the existing data pool of stored company profiles. More visionary we discuss to use a 
web crawler collecting this semantically-enriched information from company web sites representing co-operation requirements.

\section{CONCLUSIONS}

In this paper we presented ODAMY to implement an efficient partner relationship management in dynamic networked organizations. We applied ontologies to allow easy on demand and fast docking of business partners. Our investigation of virtual organizations determined key criteria for strategic fit as success factor of business networking. The key criteria were transformed into a shared conceptual model by a business community. ODAMY is implemented using KAON tool set, which allows demonstrating the described functionality. In the future we will have to pay specific attention on how to integrate the tool suite in the concrete working process and to support the automation of business partner integration as well as the continuous evolution of the underlying conceptual model.

\section{REFERENCES}

1. KAON - The Karlsruhe Ontology and Semantic Web Framework. Developer's Guide for KAON 1.2.5, February 2003, http://wim.fzi.de.

2. A. Maedche, B. Motik, L. Stojanovic, R. Studer, R. Volz. Ontologies for Enterprise Knowledge Management. Technical report. FZI Karlsruhe, Germany, Institute AIFB, University of Karlsruhe, Germany, 2001.

3. V. Iosif and Y. Sure. Exploring Potential Benefits of the Semantic Web for Virtual Organizations, EU project On-To-Knowledge (IST-1999-10132). Paper on PAKM 2002.

4. B. Motik, A. Maedche, and R. Volz, A Conceptual Modeling Approach for Semantics-Driven Enterprise Applications, Technical report, FZI, Karlsruhe, Germany, 2003

5. M. Längsfeld, P. Weiß. Virtual Organizational structures. An empirical study. Research Center of Information Technology, Karlsruhe 2000.

6. N. Blumenthal. Virtual organzational structures. Intranetwork analysis. A empirical study. Research Center of Information Technology. Karlsruhe 2001.

7. M. Wollny. ICT support in virtual organizational structures. Intranetwork analysis. A empirical study. Research Center of Information Technology. Karlsruhe 2002.

8. U. Franke, B. Hickmann. Is the Net-Broker an Entrepreneur? What Role does the Net-Broker play in Virtual Webs and Virtual Corporations? Proceedings of the 2nd International VoNet - Workshop, Zurich, September 23-24, 1999.

9. W. Saabeel, T.M. Verduijn, L. Hagdorn, K. Kumar. A model of virtual organization: a structure and processs perspective. eJOV 4 (2002) 1. http://www.virtual-organization.net/.

10. Schoop, Becks, Quix, Burwick, Engels, Jarke. Enhancing Decision and Negotiation Support in Enterprise Networks Through Semantic Web Technologies. Informatik V, RWTH Aachen, Germany, FIT Fraunhofer, Germany, Thinking Networks AG, Germany. schoop@cs.rwth-aachen.de.

11. Su, Chen, Lee. Quality Management System's Design for Virtual organizations. eJOV 3 (2001) 5. http://www.virtual-organization.net.

\footnotetext{
i ODAMY stands for 'Ontology-baseD pArtnership relationship Management methologY'

ii In this paper we use the term „virtual organization" and „business network" in the same meaning, as virtual organizations can be summarised and understood as a business network with particular characteristics.
} 